\title{
Development of Wearable Heart Sound Collection Device
}

\author{
Ximing $\operatorname{HUAI}^{1}$, Shota Notsu ${ }^{2}$, Panote Siriaraya ${ }^{4}$ \\ Noriaki Kuwahara $^{5}$ \\ Graduate School of Science and Technology \\ Kyoto Institute of Technology, Kyoto, Japan
}

\author{
Dongeun $\mathrm{Choi}^{3}$ \\ Faculty of Informatics \\ The University of Fukuchiyama \\ Kyoto, Japan
}

\begin{abstract}
In recent years, the mortality rate of cardiovascular diseases and the younger generation have attracted people's attention. At the same time, there is an increasing demand for devices that can monitor the physiological parameters of the heart. In this research, a wearable devices was designed and developed for heart sound collection. Microphones wrapped in urethane resin holders were directly fixed on the vest for heart sound collection. The device has received many positive reviews in terms of comfort. The cumulative contribution rate of the two common factors (material factor and clothing design factor) obtained through factor analysis was $75.371 \%$, which was the main factor affecting the experience of using the device. Finally, the heart sounds of 11 healthy young people were collected and input into the completed convolutional neural network for detection, and an accuracy rate of $71.3 \%$ was obtained. Therefore, it can be concluded that the device improves the user experience and has a good effect on heart sound collection and detection.
\end{abstract}

Keywords-Cardiovascular diseases; wearable devices; heart sound collection; convolutional neural network

\section{INTRODUCTION}

The number of people dying from cardiovascular disease (CVD) is steadily rising, including one-third of all deaths globally in 2019, according to a paper in the Journal of the American College of Cardiology that reviewed the total magnitude of CVD burden and trends over 30 years around the world [1]. With the current aging of the world, the mortality rate of cardiovascular diseases will continue to increase. At the same time, cardiovascular disease is no longer a patent for the elderly. Among the sick people, the ratio of middle-aged and young people is also rising, and cardiovascular diseases are getting younger. The main reason for this trend is the bad lifestyle habits of young people, such as long-term smoking, lack of sleep, unhealthy eating habits, lack of exercise, etc. [23].

As the rejuvenation of cardiovascular disease becomes more and more obvious, it has attracted widespread attention, and people's health awareness is gradually improving. At the same time, it means that the demand for medical equipment is constantly expanding, not only for medical equipment in hospitals, but also for equipment that can monitor human physiological parameters in daily life. This kind of equipment does not require the patient to stay in the hospital all the time, but it can monitor various physiological parameters of the human body in real time, reflect the health of the human body, and provide accurate basis for clinical diagnosis [4-7] for doctors to make timely diagnosis and treatment, to improve the efficiency of the hospital's diagnosis of the patient's condition. For elderly people and young people with great work pressure, getting 24 hours uninterrupted supervision in their daily life, can make them gain a sense of security to a large extent. The remote monitoring of physiological parameters has become a research hotspot in the field of biomedical engineering [8-9].

At present the type of physiological parameter monitoring equipment range on the market, divided by monitoring the way, there are wearable, portable, desktop detection equipment etc.; divided by function, there are heart sound and electrocardiogram (ECG) monitoring, defibrillation monitoring, sleep monitoring equipment etc. [10-11]. With the continuous advancement of technology and the increase of market demand, wearable monitoring equipment has become the future research direction. The most representative equipment at present is the wearable vest-type physiological parameter monitoring equipment Life Shirt developed by Vivo Metrics company in the United States. As shown in Fig. 1. The device can monitor different physiological parameters of the human body through multiple biosensors, such as ECG, blood pressure, heart rhythm, body temperature, etc. [12-14]. It has been used as a clinical medical instrument by many hospitals, but the data collected by the device cannot be transmitted in real time, so remote real-time monitoring cannot be realized.

In view of the high mortality rate of cardiovascular diseases, monitoring equipment specifically for heart sounds and ECG is also emerging in endlessly. Since heart sound auscultation is one of the most basic diagnostic methods for cardiovascular diseases, it is an important basis for the initial diagnosis [1516], so the monitoring of heart sounds can reflect the human heart condition in a timely manner. This helps doctors find abnormalities in the heart as soon as possible so as to improve the diagnosis efficiency of the disease and enable patients to receive timely treatment. In the current research on heart sound monitoring equipment, it can be roughly divided into two categories: electronic stethoscope collecting heart sounds and microphone collecting heart sounds. Among them, the development of electronic stethoscope collection of heart sounds is relatively mature. For example, Beck C et al. [17] designed and developed a multi-mode physiological parameter collection wearable device including heart sound auscultation, which can be used with MATLAB software to obtain real-time data or store data in software; Tiwari, Hemant Kumar et al. [18] have designed an embedded stethoscope served as a platform 
for the computer aided diagnosis of cardiac sound for the detection of cardiac murmur, the device can display heart sounds on the TFT LCD display in real time, and stored on the micro SD card. However, these devices are inconvenient for daily carrying due to their large size. In terms of microphone collection of heart sounds, Kirchner J et al. [19] proposed a wearable system for long-term capture of chest sound, including four microphones for receiving physiological signals and one microphone for receiving ambient noise. The performance of the device was evaluated in different environments, and the use of combined microphones improved the noise cancellation; Aguilera-Astudillo C et al. [20] demonstrate a chest piece which consists of an electret microphone embedded into the drum of a $3 \mathrm{D}$ printed chest piece. And use the electronic dongle to amplify the microphone signal and reduce external noise, while the mobile phone can display the heart sound signal. From the above studies, we can find that the use of electronic stethoscopes to collect heart sounds is not convenient for daily wear due to the size of electronic stethoscopes, and the use of microphones to collect heart sounds did not investigate the comfort of the device and the wearing experience, so we believe that there is still more room for research in the wearing comfort of heart sound monitoring devices.

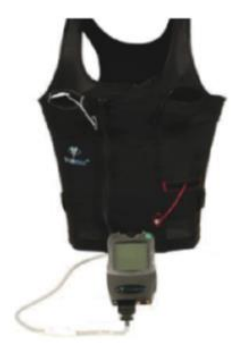

Fig. 1. Life Shirt Wearable Physiological Parameter Monitoring System.

In view of previous research, this paper is devoted to the design and development of an easy-to-wear heart sound collection device, to discuss and study the comfort of the device, and to analyze the collected heart sounds using convolutional neural networks $(\mathrm{CNN})$. The organization structure of this article is as follows: Section II explains the design of the heart sound collection vest in detail; Section III introduces the heart sound collection experiment and heart sound data analysis; Section IV analyzes the results and discusses them; Section V draws conclusions and Section VI summarizes the shortcomings and makes suggestions for future work.

\section{Design OF THE HeART Sound Collection Vest}

This part introduces the design and development of the heart sound collection vest. First, a 3D printing mold was designed and printed according to the size of the microphone. After that, urethane resin was used to make a super soft microphone holder. Finally, according to the 4 positions of the heart sound auscultation, the microphone was fixed on the vest to make a wearable heart sound collection vest.

\section{A. The Design of the Microphone Holder}

1) $3 D$ printing mold design: The microphone used in this design is the Uni-directional Electret Condenser Microphone produced by Primo Company, the model is EM297, as shown in Fig. 2, the diameter is $10 \mathrm{~mm}$, and the thickness is $4.5 \mathrm{~mm}$. According to the instructions of the microphone, the sound hole on the back of the microphone cannot be blocked in order to receive the sound. At the same time, in order to ensure that the microphone can accurately receive the sound, the microphone needs to be close to the skin, which requires the microphone to withstand a certain amount of pressure, so it is necessary to make a suitable holder to ensure that the microphone can receive normally and accurately.

According to [21], when the diameter of the holder is $17 \mathrm{~mm}$, and there is a $1.5 \mathrm{~mm}$ thick silicone layer between the microphone and the skin, the microphone has the best sound reception. Therefore, according to the shape and size of the microphone, the 3D printing mold of the holder has shown in Fig. 3 was designed. The mold is a cylinder and adopts a fullywrapped type except for the sound hole on the back. At the same time, a dedicated space is reserved for the microphone wire.

2) Development of soft skin-friendly holder: In view of the fact that the microphone holder needs to be in direct contact with the skin, the urethane resin with a hardness of 0 produced by EXSEAL company was selected to make the holder in consideration of skin affinity when selecting the material. This urethane resin has excellent softness, sufficient molding strength even if the hardness is 0 , and this urethane resin has a softness like a human skin.
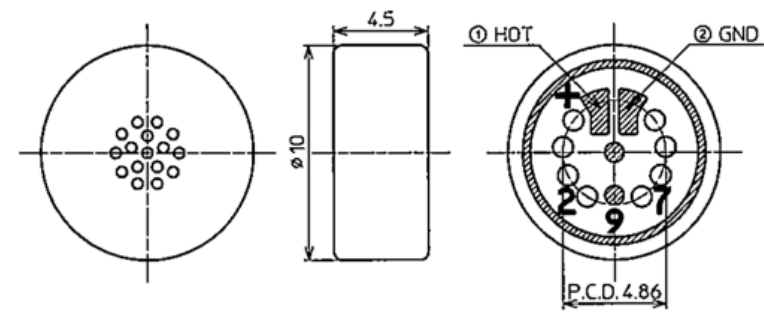

Fig. 2. Schematic Diagram of Microphone.
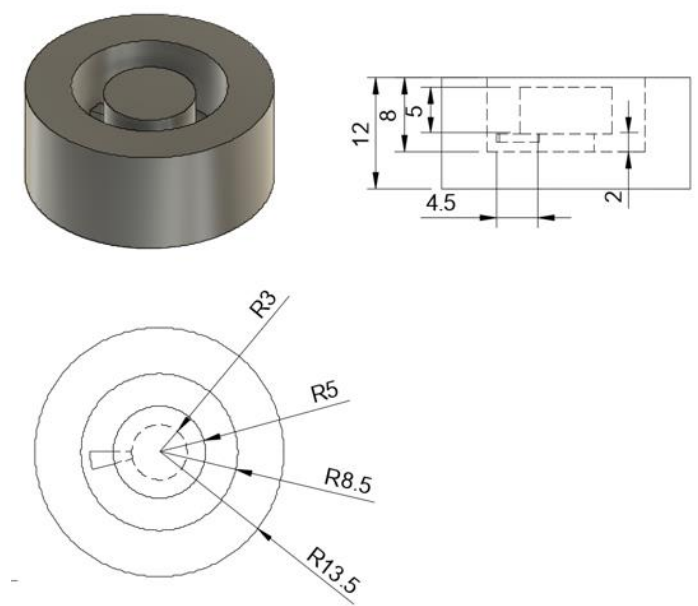

Fig. 3. Schematic Diagram of the 3D Printing Model of the Microphone Holder (unit: mm) (a) Holder Molding Model (b) Model Front view (c) Model Top View. 
According to the product's instructions, first calculate the volume of the mold before preparing the gel, and spray the mold with a special release agent for urethane resin in advance to facilitate the release of the holder afterwards; then mix the two liquids (the weight ratio of main agent and curing agent is $3: 1$ ) and pour them into the 3D printing mold; finally, after 24 hours, take out the molded holder from the mold. Fig. 4 shows the process of manufacturing the microphone holder. The holder wraps the microphone well and protects the relatively fragile and breakable wires of the microphone. Fig. 5 is a schematic diagram after the microphone is installed in the holder.

\section{B. The Design of the Heart Sound Collection Vest}

1) Location of heart sound auscultation: According to [22], there are 4 commonly used positions for heart sound auscultation. As shown in Fig. 6, the mitral valve is located at the strongest point of the apical beat, and is normally located at the fifth intercostal space on the inner side of the left midclavicular line; the pulmonary valve is in the second intercostal space on the left edge of the sternum; the aortic valve is in the second intercostal space on the right edge of the sternum; the tricuspid valve is on the left edge of the lower end of the sternum, that is, the 4-5th intercostal space on the left edge of the sternum. The general auscultation sequence is the beginning of the mitral valve - the pulmonary valve - the aortic valve - the tricuspid valve.

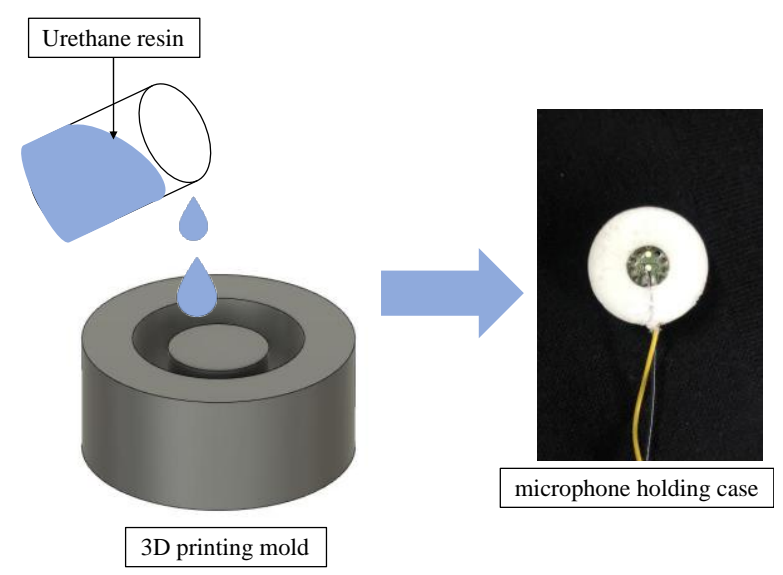

Fig. 4. Schematic Diagram of Making Microphone Holder.

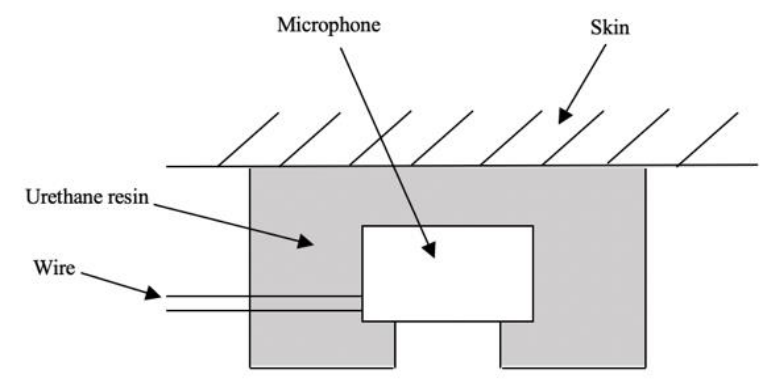

Fig. 5. Schematic Diagram of the Microphone Placed in the Holder.

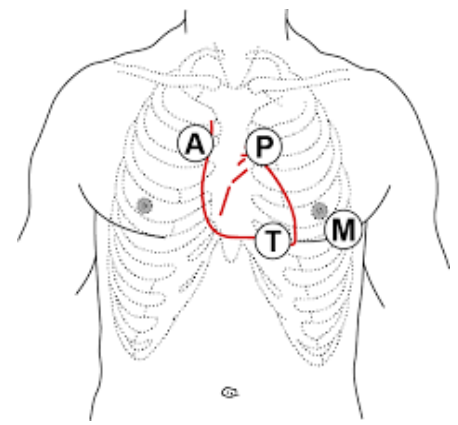

Fig. 6. Position of Heart Sound Auscultation. $\mathrm{A}=$ Aortic Valve; $\mathrm{P}=$ Pulmonic Valve; $\mathrm{M}=$ Mitral Valve; $\mathrm{T}=$ Tricuspid Valve.

2) Making of heart sound collection vest: A wearable vest for heart sound acquisition was proposed as the main object of this study based on the location of heart sound auscultation and taking into account the comfort, fit and appearance of the device.

A fabric made of Polyester $90 \%$ and Lycra $10 \%$ was used as the base fabric, and a suitable vest was made according to the $\mathrm{m}$ size of men's and women's clothing size. The vest was designed with two layers of fabric, which could facilitate the fixation of the microphone and make the vest neat in appearance. After that, according to the four positions of heart sound auscultation, the fixing sleeve with microphone is fixed on the corresponding position on the inside of the vest: firstly, the position of the microphone is determined, then it is wrapped with cloth and sewn on the vest, then holes are made at the suitable position nearby for the wire to pass through, and finally the wire is fixed on the vest with needle and thread and passed out from the side of the vest. As shown in Fig. 7, (a) is a diagram of the microphone device inside the vest, and (b) is a diagram of the exterior of the vest.

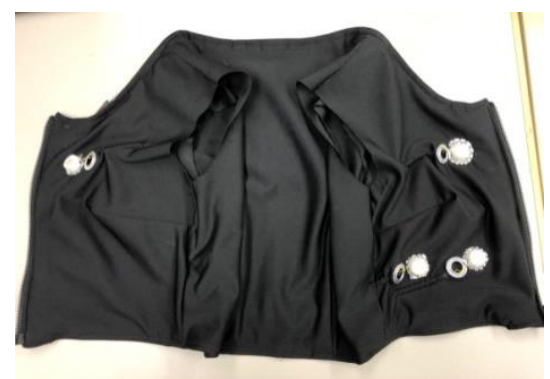

(a)

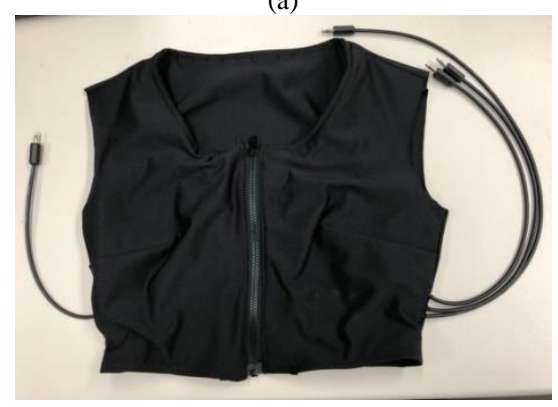

(b)

Fig. 7. Physical Diagram of the Heart Sound Acquisition Vest (a) Microphone Device on the Inside of the Vest (b) Diagram of the Outside of the Vest. 


\section{EXPERIMENTS AND METHODS}

This section describes the experiments used to evaluate the acquisition effectiveness of the designed heart sound collection vest and the comfort of wearing it.

\section{A. Heart Sound Collection Experiment}

A heart sound collection experiment was conducted on healthy young people to evaluate the collection effect and wearing comfort of the heart sound collection vest. 11 students (5 females and 6 males) participated in the experiment. Ages ranged from 21 to 29 . Height, weight and chest circumference of the participants were measured before the experiment: height ranged from $1.61-1.7 \mathrm{~m}$, weight ranged from $54-56 \mathrm{~kg}$ and chest circumference ranged from $80-93 \mathrm{~cm}$ for females, and height ranged from 1.58-1.75m, weight ranged from 55-68 $\mathrm{kg}$ and chest circumference ranged from $81-98 \mathrm{~cm}$ for males.

The heart sound collection experiment was carried out in an indoor experimental environment with a temperature of 20 degrees Celsius, a humidity of $45 \%$, and a surrounding environment with a decibel number of $15 \mathrm{~dB}$. The participants wore a vest of appropriate size, and the heart sounds were collected and stored in the following order of auscultation: mitral valve area - pulmonary valve area - aortic valve area tricuspid valve area, with 3-5 heart sounds randomly collected for 30-180 seconds at each location, as shown in Fig. 8..

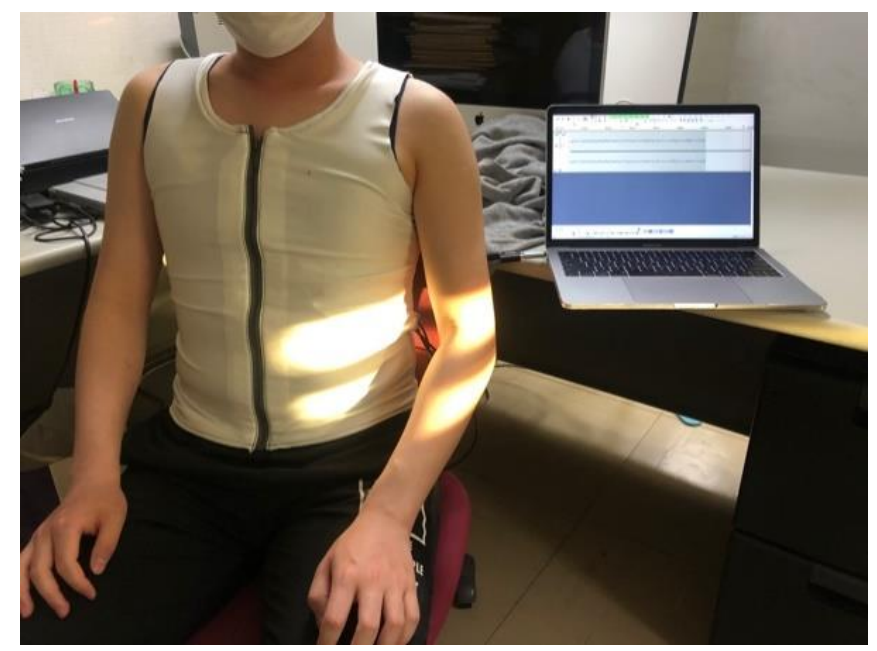

Fig. 8. Experimenter in Heart Sound Collection Experiment

\section{B. Investigation of Device Comfort}

After the completion of the heart sound collection experiment, the experimental participants were asked to complete an assessment questionnaire for the heart sound collection vest. The questionnaire was set up with reference to the Semantic Difference [23], which is a measure of semantic differentiation. It was developed by the social psychologist Osgood et al. in the 1950s. Such scales consist of a series of bipolar adjective word pairs that are generally divided into seven equal rating scales. They have the quality of showing the semantic space in which any concept has meaning, and can be used accordingly to describe the underlying meaning of any concept and its associated problematic nature or property aspects. In the present study for the wearable device, considering the user-friendly characteristics that the device needs to have, seven contrasting adjectives and a 7-point rating scale from -3 to +3 were proposed in terms of material, prolonged wear, and appearance for the experimental participants to rate this heart sound collection vest. The seven adjectives were 1) tight/loose, 2). Strong/weak, 3) heavy/light, 4) hard/soft, 5) unattractive/nice, 6) inconvenient/convenient, 7) dislike/like. Each adjective group corresponds to an explanatory question: 1) Do you think the pressure exerted by this device on the skin is tight or loose? 2) Do you think the stimulation of this device on the skin is strong or weak? 3) Do you think this device is heavy? 4) Do you think the touch of this device is hard or soft? 5) Do you think the design of this device is good-looking? 6) Do you think this device is convenient to put on and take off? 7) Do you like to use this device for a long time?

\section{RESULTS AND DISCUSSION}

\section{A. Heart Sound Data Denoising}

There are many noises generated during heart sound collection, for example: ambient noise; vibration of the microphone caused by chest vibration and the zipper of the vest is located in the middle of the chest causing the microphone in the pulmonary valve area and aortic valve area to not fit well to the skin, generating a lot of noise, etc. Therefore, filtering and denoising are needed before heart sound data detection. In this study, an algorithm written in python is used to remove the noise. The steps of the algorithm are: 1) calculate Fast Fourier Transform (FFT) on the noisy audio fragment, 2) calculate statistics from the FFT of the noise, 3) calculate a threshold based on the statistics of the noise (and the desired sensitivity of the algorithm), 4) calculate FFT from the signal, 5) determine the mask by comparing the signal FFT with the threshold, 6) the mask is smoothed in frequency and time using a filter, 7) the mask is applied to the FFT of the signal and is inverted. After processing the heart tone data using this algorithm, the spectrograms of the heart tone data before and after denoising are obtained as shown in Fig. 9. The upper panel is before denoising and the lower panel is after denoising, and it can be clearly seen that most of the noise is removed.

\begin{tabular}{|c|c|c|c|c|c|c|}
\hline 0.0 & 0.5 & 1.0 & 1.5 & 20 & 2.5 & 3.0 \\
\hline $\begin{array}{c}20000 \\
15000 \\
10000 \\
7000 \\
5000 \\
3000 \\
2000 \\
1000 \\
600 \\
600\end{array}$ & & & & & & \\
\hline \begin{tabular}{|c|}
20000 \\
14000 \\
10000 \\
77000 \\
5000 \\
3000 \\
2000 \\
1000 \\
500 \\
100 \\
100
\end{tabular} & & & & & & \\
\hline
\end{tabular}

Fig. 9. Spectrograms of Heart Sounds before and after Denoising.

\section{B. Heart Sound Data Detection Results}

To test the denoising effect, the heart sound data before and after denoising were fed into the convolutional neural network (CNN) [24] that had been built to obtain the accuracy of heart sound data detection. Firstly, the heart sound data were divided 
in 5-second intervals, and then the sample heart sounds were converted into grayscale spectrograms by an automatic procedure using the librosa library, and finally fed into the CNN. This CNN consists of 5 layers, including 3 convolutional layers, 1 fully connected layer and 1 normalized exponential function softmax classification layer. The network parameters for each layer are as follows: for the first convolutional layer, we used 32 filters with a convolutional kernel size of $3 \times 3$ and a step size of $1 \times 1$, and the pooling (using the max-pooling method) size was $2 \times 2$ and a step size of $1 \times 1$. We used a modified linear unit (ReLU) activation function with a random deactivation (loss) rate of 0.1 . For the second and third convolutional layers, we used 64, 128 filters. The convolution kernel size is $3 \times 3$ and the step size is $1 \times 1$. The pooling operation, activation function and dropout probability were the same as in the previous layer. For the fourth layer, we use a fully connected dense layer with an output size of 500, a ReLU activation function and a dropout function (probability 0.25 ). In the last classification layer, we used a Softmax classifier.

The detection of the convolutional neural network shows that the accuracy of the un-denoised heart sound data is $34.89 \%$ and the accuracy of the denoised heart sound data is $71.30 \%$. In order to investigate the detection accuracy of the heart sound collection vest under the wearing of different gender groups, the detection results of the heart sound data were analyzed according to male and female as well as each person, as shown in Tables I, II, and III.

From Table I, it can be seen that although the accuracy of heart sound data for males before denoising was lower than that of heart sound data for females, after denoising, the accuracy of heart sound data for both males and females exceeded $70 \%$, which indicates that the denoising procedure was effective and was able to remove most of the noise and improve the accuracy of heart sound detection.

Table II shows the comparison of the accuracy of heart sound data before and after denoising for males, from which it can be found that the accuracy of the heart sound data obtained in the state of collecting heart sounds while wearing the same vest varies due to the different height, weight and chest circumference of each experimental participant. The reason for this is that the tighter the vest fits to the skin, the clearer the heart sound data is and the higher the accuracy rate is after denoising. Therefore, the better the fit of the vest to the skin, the better the collection results.

Table III shows the comparison of the accuracy of heart sound data before and after denoising in women, from which it can be found that the heart sound data of experimental participants No. 1 and No. 2 obtained more than 91\% accuracy after denoising, and the other three also obtained more than $64 \%$ accuracy, indicating that the heart sound acquisition vest can acquire heart sounds well. However, the detection accuracy of individual heart sound data varied widely due to the difference in body size of each individual and the fact that the vest for women was designed without the inclusion of a bra part.

\section{Factor Analysis of Heart Sound Collection Vest}

Fig. 10 visualizes the semantic difference (SD) evaluation scores of men and women on wearable heart sound collection devices. It can be seen from the figure that men and women have roughly the same evaluation of the device, but women have given more positive feedback. Among them, both men and women think that the device has weak skin irritation, not heavy, soft, convenient to wear, and suitable for long-term use. These positive feedbacks may be attributed to the following factors: 1) Polyester and lycra used in the fabric of the vest are soft and stretchable, suitable for personal wear; 2) The microphone holder is made of urethane resin with a hardness of 0, it has a softness like a human skin; 3) The vest is designed with a zipper to facilitate putting on and taking off. At the same time, women think that the device has little pressure on the skin and the appearance is attractive, but men think that the device has a certain amount of pressure on the skin and the appearance is not very attractive. This is because there are certain differences in the design of the male and female vests, and it is also the direction of improvement in the future.

TABLE I. COMPARISON OF THE ACCURACY OF HEART SOUND DATA DETECTION BEFORE AND AFTER DENOISING

\begin{tabular}{|l|l|l|}
\hline & $\begin{array}{l}\text { Accuracy of data without } \\
\text { denoising }\end{array}$ & $\begin{array}{l}\text { Accuracy of data after } \\
\text { denoising }\end{array}$ \\
\hline Male & $27.36 \%$ & $70.01 \%$ \\
\hline Female & $40.16 \%$ & $72.20 \%$ \\
\hline
\end{tabular}

TABLE II. COMPARISON OF THE ACCURACY OF HEART SOUND DATA DETECTION BEFORE AND AFTER DENOISING FOR MALES

\begin{tabular}{|l|l|l|l|l|l|}
\hline & $\begin{array}{l}\text { Height } \\
\text { (cm) }\end{array}$ & $\begin{array}{l}\text { Weight } \\
\text { (kg) }\end{array}$ & $\begin{array}{l}\text { Chest } \\
\text { circumference } \\
\text { (cm) }\end{array}$ & $\begin{array}{l}\text { Accuracy of } \\
\text { data } \\
\text { without } \\
\text { denoising }\end{array}$ & $\begin{array}{l}\text { Accuracy } \\
\text { of } \\
\text { denoised } \\
\text { data }\end{array}$ \\
\hline M-1 & 168 & 68 & 95 & $42.54 \%$ & $85.39 \%$ \\
\hline M-2 & 173 & 67.5 & 98 & $43.51 \%$ & $83.76 \%$ \\
\hline M-3 & 175 & 65 & 87 & $11.28 \%$ & $72.16 \%$ \\
\hline M-4 & 175 & 60 & 89 & $12.96 \%$ & $70.15 \%$ \\
\hline M-5 & 167 & 55 & 81 & $12.16 \%$ & $55.32 \%$ \\
\hline M-6 & 158 & 58 & 86 & $41.35 \%$ & $54.31 \%$ \\
\hline
\end{tabular}

TABLE III. COMPARISON OF THE ACCURACY OF HEART SOUND DATA DETECTION BEFORE AND AFTER DENOISING FOR FEMALES

\begin{tabular}{|l|l|l|l|l|l|}
\hline & $\begin{array}{l}\text { Height } \\
(\mathbf{m})\end{array}$ & $\begin{array}{l}\text { Weight } \\
(\mathbf{k g})\end{array}$ & $\begin{array}{l}\text { Chest } \\
\text { circumference } \\
(\mathbf{c m})\end{array}$ & $\begin{array}{l}\text { Accuracy of } \\
\text { data } \\
\text { without } \\
\text { denoising }\end{array}$ & $\begin{array}{l}\text { Accuracy of } \\
\text { denoised } \\
\text { data }\end{array}$ \\
\hline F -1 & 162 & 55.6 & 90 & $24.20 \%$ & $91.59 \%$ \\
\hline F-2 & 170 & 55 & 80 & $44.95 \%$ & $91.57 \%$ \\
\hline F-3 & 163 & 55 & 92.5 & $31.18 \%$ & $68.25 \%$ \\
\hline F -4 & 161 & 54 & 85 & $51.95 \%$ & $66.67 \%$ \\
\hline F -5 & 169 & 56 & 82 & $64.73 \%$ & $64.87 \%$ \\
\hline
\end{tabular}




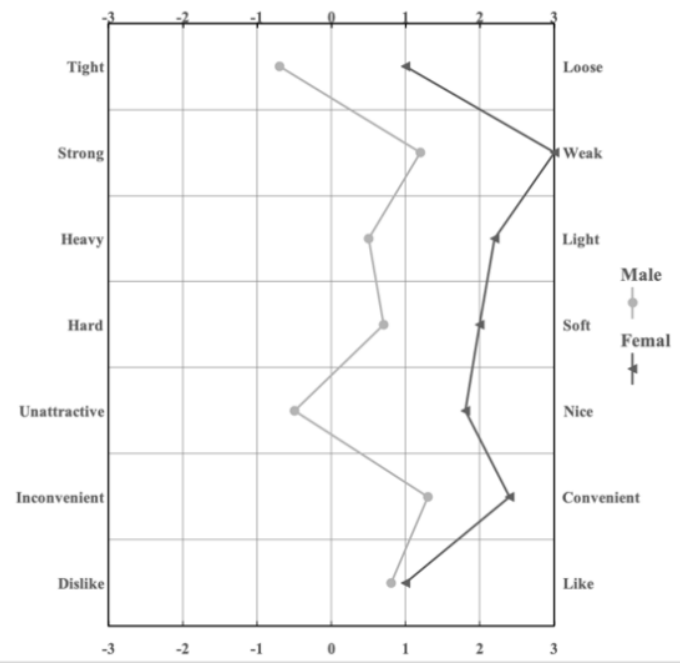

Fig. 10. Image Scale of SD Evaluation Score (Average Value) of Male and Female Wearable Heart Sound Acquisition Devices.

Factor analysis was conducted on the SD scores of 11 experimental participants on the wearable heart sound collection vest [25]. Factor analysis is the conversion of multiple measured variables into a small number of composite indicators (or latent variables), and it reflects an idea of dimensionality reduction. The variables with high correlation are clustered together through dimensionality reduction, thus reducing the number of variables that need to be analyzed while reducing the complexity of the problem analysis. Thus, the use of factor analysis provides insight into the potential factors affecting wearable heart sound collection devices.

Factor analysis was conducted using python. First, to confirm the suitability of the data for factor analysis, KaiserMeyer-Olkin (KMO) and Bartlett tests were performed with: $\mathrm{KMO}$ test is used to examine the bias correlation between variables, taking values between 0 and 1 ; the closer the KMO statistic is to 1 , the stronger the bias correlation between variables and the better the factor analysis. Generally the statistic is above 0.6 is adapted to do factor analysis [26]; and the smaller the value of P-Value indicates the more significant data differences. The results obtained through the test are shown in Table IV, the KMO of this experimental data $=0.617>$ $0.6, \mathrm{P}-$ Value $=0.001$, so it is suitable for factor analysis.
Then, the scree plot was drawn according to the number of factors and the corresponding eigenvalues of each factor, as shown in Fig. 11; Table V shows the total variance explained for each component of the factor analysis of the heart sound collection vest. From Fig. 11 and Table V, it can be obtained that the eigenvalues of the first 2 factors are greater than 1 , while the variance contribution of these 2 common factors accounts for $75.371 \%$, indicating that the extraction of these 2 common factors can explain the majority of the information of the original data. From Fig. 11 and Table V, it can be obtained that the eigenvalues of the first 2 factors are greater than 1 , while the variance contribution of these 2 common factors accounts for $75.371 \%$, indicating that the extraction of these 2 common factors can explain the majority of the information of the original data. Therefore, the number of common factors was determined as 2 to perform the required factor extraction.

TABLE IV. THE KMO AND BARLETT'S TEST OF THE FACTOR ANALYSIS OF THE WEARABLE DEVICES

\begin{tabular}{|l|l|l|}
\hline \multicolumn{2}{|l|}{ Kaiser-Meyer-Olkin Measure of Sampling Adequacy. } & 0.617 \\
\hline \multirow{2}{*}{ Bartlett's Test of Sphericity } & Approx. Chi-Square & 41.853 \\
\cline { 2 - 3 } & Sig.(P-Value) & 0.001 \\
\hline
\end{tabular}

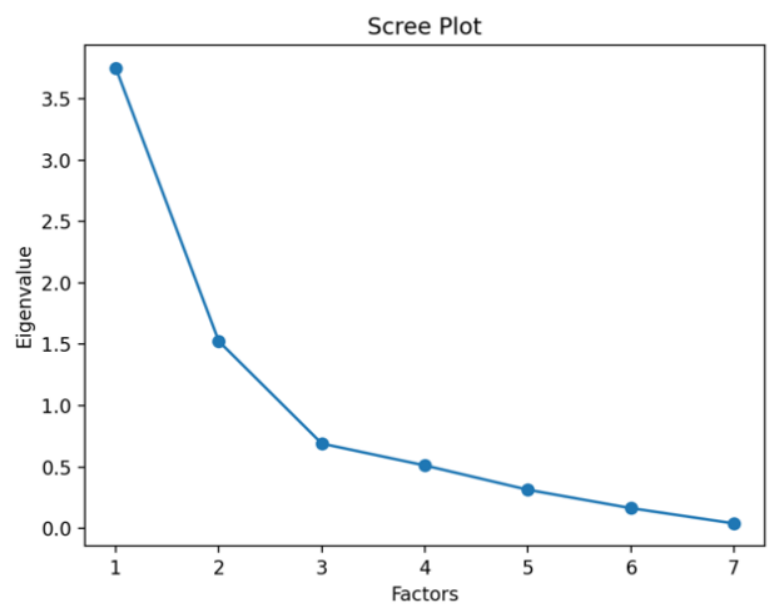

Fig. 11. Scree Plot of the Factor Analysis of the Heart Sound Collection Vest Evaluation.

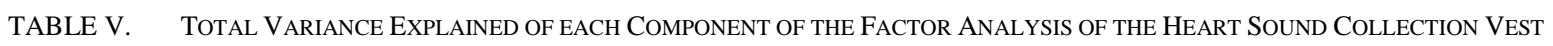

\begin{tabular}{|l|l|l|l|l|l|l|}
\hline & \multicolumn{2}{|l|}{ Initial Eigenvalues } & \multicolumn{3}{l|}{ Rotation Sums of Square Loadings } \\
\hline Component & Total & of Variance \% & Cumulative \% & Total & of Variance \% & Cumulative \% \\
\hline 1 & 3,751 & 53.578 & 53.578 & 3.499 & 49.983 & 49.983 \\
\hline 2 & 1.525 & 21.783 & 75.361 & 1.777 & 25.388 & 75.371 \\
\hline 3 & 0.691 & 9.870 & 85.231 & & & \\
\hline 4 & 0.513 & 7.328 & 92.559 & & & \\
\hline 5 & 0.315 & 4.499 & 97.058 & & & \\
\hline 6 & 0.165 & 2.357 & 99.415 & & & \\
\hline 7 & 0.041 & 0.586 & 100.000 & & & \\
\hline
\end{tabular}


TABLE VI. THE ROTATED COMPONENT MATRIX OF THE FACTOR ANALYSIS

\begin{tabular}{|l|l|l|}
\hline & Factor1 & Factor2 \\
\hline Heavy - Light & 0.932 & 0.151 \\
\hline Unattractive - Nice & 0.888 & 0.418 \\
\hline Strong - Week & 0.811 & 0.011 \\
\hline Tight - Loose & 0.795 & 0.271 \\
\hline Hard - Soft & 0.733 & -0.222 \\
\hline Inconvenient - Conveniente & 0.053 & 0.864 \\
\hline Dislike - Like & 0.102 & 0.843 \\
\hline
\end{tabular}

Finally, the results shown in Table VI were obtained after rotating the factor loading matrix according to the method of great variance. Through Table VI, it can be found that common factor 1 has larger loadings on factors 1-5 (light pressure; weak skin irritation; lightweight; soft; good-looking), and common factor 2 has larger loadings on factors 6 and 7(easy to put on and take off, preferring long-term use), indicating that these 7 variables can be reduced to 2 male factors: material factor and garment design factor.

Compared with previous studies [17-20], this research designed and developed a complete wearable device instead of a single heart sound collector, and explored the wearing experience of the heart sound collection device, and the experimental participants gave a positive evaluation, all of whom found that the device is suitable for daily wear. In consistent with them the denoised heart sounds were clear, and additionally heart sound detection was added, using a convolutional neural network system to detect the pairs of collected heart sounds and obtain better results.

\section{CONCLUSION}

In this paper, a comfortable wearable heart sound acquisition device is presented. First, a urethane resin holder was fabricated according to the size and characteristics of the microphone. Then, suitable materials were selected and vests were made according to the physical characteristics of men and women respectively. Then, the microphone wrapped with holder was fixed on the corresponding position of the vest with reference to the heart sound auscultation position. Subsequently, 11 healthy young people ( 6 males and 5 females) were subjected to heart sound collection, and the collected heart sounds were input into the constructed $\mathrm{CNN}$ for testing to evaluate the performance of the device, and the highest accuracy rate of $85.39 \%$ for a single male, the highest accuracy rate of $91.59 \%$ for a single female and an average accuracy rate of $71.3 \%$ were obtained. Finally, the evaluation of the device by the experimental participants was analyzed and 2 common factors were extracted: material factor and garment design factor, indicating that these two factors can be focused on for future updates of the device. This study demonstrates that the wearable heart sound collection device is effective in heart sound collection and has been well evaluated in terms of product design.

\section{FUTURE WORK}

The wearable heart sound collection device developed in this study can be used well for heart sound collection, but there are still many shortcomings. First, the design of the zipper in the middle of the chest causes a lot of noise during heartbeat collection, and the position of the zipper needs to be reconsidered and adjusted; second, the position of the microphone cannot be adjusted according to the size of each person and needs to be redesigned; third, a more skin-friendly material could be selected; and finally, the data transmission of the device should be updated to wireless transmission. It is hoped that in the future, a real-time heartbeat detection device can be made that can be worn daily for a long time.

\section{ACKNOWLEDGMENT}

This research was supported by JSPS KAKENHI Grant Number 19H04154. We would also like to thank the China Scholarship Council (CSC) for supporting the research of PhD student Huai Ximing, and to teachers and experiment participants who assisted in completing this research.

\section{REFERENCES}

[1] Roth, G. A., Mensah, G. A., Johnson, C. O., Addolorato, G., Ammirati, E., Baddour, L. M., ... \& GBD-NHLBI-JACC Global Burden of Cardiovascular Diseases Writing Group, "Global burden of cardiovascular diseases and risk factors, 1990-2019: update from the GBD 2019 study," Journal of the American College of Cardiology, vol. 76(25), 2982-3021, 2020.

[2] W. Herrington, B. Lacey, P. Sherliker, J. Armitage and S. Lewington, "Epidemiology of atherosclerosis and the potential to reduce the global burden of atherothrombotic disease," Circulation research, vol.118(4), pp.535-546, 2016.

[3] M. Nahrendorf and F. K. Swirski, "Lifestyle effects on hematopoiesis and atherosclerosis," Circulation research, Vol.116(5), pp.884-894,2015.

[4] P. Pandian, K. Mohanavelu, K. Safeer, T. Kotresh, D. Shakunthala, P. Gopal, et al. "Smart Vest: Wearable multi-parameter remote physiological monitoring system," Medical engineering \& physics, vol.30(4), pp.466-477,2008.

[5] Z. Xu, Z. Fang, L. Du, Z. Zhao, X. Chen, D. Chen, et al. "A Wearable Multi-parameter Physiological System," In Ubiquitous Information Technologies and Applications, pp. 643-648, Springer, Berlin, Heidelberg, 2014.

[6] C. Yu, Q. He, R. Li, J. TAN and L. YU, "Research of Multiple Physiological Parameters Monitoring System for Sub-healthy Groups," Piezoelectrics and Acoustooptics, vol.35(1), pp.136-139, 2013.

[7] J. Welch, J. Moon and S. McCombie, "Early detection of the deteriorating patient: the case for a multi-parameter patient-worn monitor," Biomedical instrumentation \& technology, vol.46(s2), pp. 5764, 2012.

[8] S. Dai and Y. Zhang, "A wireless physiological multi-parameter monitoring system based on mobile communication networks," In 19th IEEE Symposium on Computer-Based Medical Systems (CBMS'06), IEEE, pp. 473-478, 2006.

[9] M. Cavalleri, R. Morstabilini and G. Reni, "Integrating telemonitoring with clinical informationsystems: a case study," Proceedings of the 2005 IEEE engineering in medicine and biology 27 th annual conference. Shanghai: IEEE, pp.573 - 576, 2005.

[10] A. Pantelopoulos and N. G. Bourbakis, "A survey on wearable sensorbased systems for health monitoring and prognosis," IEEE Transactions on Systems, Man, and Cybernetics, Part C (Applications and Reviews),vol.40(1),pp.1 - 12, 2010.

[11] A. Lmberis and A. Dittmar, "Advanced wearable health systems and applications - research and development efforts in the European Union," IEEE Engineering in Medicine and Biology Magazine, vol.26(3), pp.29 $-33,2007$. 
[12] P. Grossman, "The LifeShirt: a multi-function ambulatory system monitoring health, disease, and medical intervention in the real world," Studies in Health Technology and Informatics, vol.108, pp.133 -141, 2004.

[13] F. H. Wilhelm, W. T. Roth and M. A. Sackner, "The LifeShirt: an advanced system for ambulatory measurement of respiratory and cardiac function," Behavior Modification, vol.27(5), pp.671 - 691, 2003.

[14] HC. A. Hollier, A. R. Harmer, L. J. Maxwell, C. Menadue, G. N. Willson, D. A. Black, et al. "Validation of respiratory inductive plethysmography(LifeShirt) in obesity hypoventilation syndrome," Respiratory Physiology \& Neurobiology, vol.194, pp.15 - 22, 2014.

[15] D. Roy, J. Sargeant, J. Gray, B. Hoyt, M. Allen and M. Fleming, "Helping family physicians improve their cardiac auscultation skills with an interactive cd-rom," Journal of Continuing Education in the Health Professions, vol.22, pp. 152-159, 2002.

[16] T.-h. CHEN, L.-q. HAN, H.-t. TANG and R.-j. ZHENG, "Research on Analysis Method and Application of Heart Sound Signals," Journal ofBeijing Technology and Business University (Natural Science Edition), vol.27(2), pp. 35-39, 2009.

[17] C. Beck and J. Georgiou, "Wearable, multimodal, vitals acquisition unit for intelligent field triage." Healthcare technology letters, vol.3(3), pp.189-196, 2016.

[18] H. K. Tiwari and A. Harsola, "Development of embedded stethoscope for Heart Sound." 2016 International Conference on Wireless Communications, Signal Processing and Networking (WiSPNET). IEEE, pp.1547-1551, 2016.
[19] J. Kirchner, S. Souilem and G. Fischer, "Wearable system for measurement of thoracic sounds with a microphone array." 2017 IEEE SENSORS. IEEE, pp.1-3, 2017.

[20] C. Aguilera-Astudillo, M. Chavez-Campos, A. Gonzalez-Suarez and J. L. Garcia-Cordero, "A low-cost 3-D printed stethoscope connected to a smartphone." 2016 38th Annual International Conference of the IEEE Engineering in Medicine and Biology Society (EMBC). IEEE, pp.43654368, 2016.

[21] S. Shimizu, M. Otani and T. Hirahara, "Frequency characteristics of several non-audible murmur (NAM) microphones." Acoustical science and technology, vol.30(2), pp.139-142, 2009.

[22] B. Karnath and W. Thornton, "Auscultation of the heart." Hospital Physician, vol.38(9), pp.39-45, 2002.

[23] L. Feng, B. An, "Partial Label Learning by Semantic Difference Maximization," IJCAI ,pp. 2294-2300, 2019.

[24] X. Huai, S. Kitada, D. Choi, P. Siriaraya, N. Kuwahara and T. Ashihara, "Heart sound recognition technology based on convolutional neural network," Informatics for Health and Social Care, pp.1-13, 2021.

[25] T. A. Brown, "Confirmatory factor analysis for applied research," Guilford publications, 2015.

[26] A. G. Yong and S. Pearce, "A beginner's guide to factor analysis: Focusing on exploratory factor analysis," Tutorials in quantitative methods for psychology, vol. 9(2), pp. 79-94, 2013. 